\title{
Printing and Prototyping of Tissues and Scaffolds
}

Brian Derby

School of Materials, University of Manchester

Oxford Road, Manchester M13 9PL, UK.

\section{Introduction}

The development of implantable medical devices and organ transplantation has radically changed the scope for medical intervention to deal with chronic medical problems and potential end of life situations. However, issues of device failure, the limited supply of donor organs and the need for immunosuppresants are ongoing problems. The broad areas of tissue engineering and regenerative medicine look to solve some of the problems though the augmentation of the healing process or the fabrication of autologous tissue grafts that will not cause an immune response in the host. It has been suggested that we will be able to build complete organs from scratch and the term organ printing has been coined and used by the popular press. Although such a goal is clearly only likely to be attainable in the far future, printing and related technologies are of current use in the area of tissue engineering and regenerative medicine.

An important concept in tissue engineering is the scaffold. Cells, donated by the patient, are expanded in culture. These are then transferred to a 3-dimensional highly porous substrate, the scaffold. The scaffold provides a surface on which cells adhere, thrive, multiply and generate the extra-cellular matrix (ECM) of structural and functional proteins and saccharides that make up living tissue. Both the scaffold material composition and its internal architecture (dimensions of the struts, walls, pores or channels) control the behaviour and well-being of the cells seeded inside. Within the body cells must be very close to the capillary network that supplies oxygen and nutrients. Similarly, within a tissue scaffold all cells must be supplied with the means to maintain life and this is achieved initially by providing a highly porous open structure to allow the uninterrupted flow and access of culture media in a bioreactor. In many ways the engineering component of tissue engineering lies in the design and manufacture of the scaffold. Hutchmacher (1) gave an early analysis of the topological requirements of a suitable scaffold material and reviewed the manufacturing routes that could be used to achieve the desired structure. Traditionally, porous scaffolds have been made by a number of routes that result in a foam-like internal structure with a random architecture with a limited control of scale. However the development of rapid prototyping techniques since the 1980s has enabled our ability to fabricate the desired, complex fine scale internal porous structure, allowing a true engineering of the scaffold. $(1,2)$

Rapid prototyping methods, sometimes referred to as 3D manufacturing or solid free form manufacture, produce complex objects from a 3-Dimensional design file by decomposing the object's shape into a series of parallel slices. The shape is then produced by reproducing these slices by laying down material a layer at a time, building up the structure. The philosophy of the method is to create objects by adding material, hence it is now generally referred to as additive manufacture (AM), to distinguish it from conventional machining, which removes material in a subtractive manner. With design files now held in digital format as standard, it is relatively easy to convert an 
arbitrary object into the slices required by AM fabrication tools. These slice design files are used to generate solid layers using a range of manufacturing techniques including: selective polymerisation, selective sintering/melting, building solids through the laying down of viscous threads and 3dimensional inkjet printing.

These new manufacturing tools have recently attracted extensive interest in the media as offering a new paradigm for distributed manufacturing (A Third Industrial Revolution: Editorial, Economist, April 21, 2012). These techniques have been used to manufacture laboratory components (3) or cell microwells (4) to order. The hardware cost for these fabrication tools has also decreased dramatically and open source equipment is now available from a number of groups. $(5,6)$ Other workers have "hacked" commercial desktop printers to fabricate objects in 2 and 3-dimensions using engineering and biological materials(7-10). These techniques, and their applications in tissue engineering, have been reviewed extensively. $(1,11)$ Early scaffolds were fabricated by AM from a single biocompatible material. It is now possible to engineer materials that contain biomimetic components to control the cellular environment. $(12,13)$ The scaffold should be a temporary feature and disappear, through dissolution or degradation, as the cells produce the ECM that defines the tissue. The materials that make up the scaffold have to be designed to fulfil this function and be compatible with the fabrication route.

Another approach to tissue engineering has been proposed that does not require a solid scaffold structure. Some workers have championed what has been identified as bottom-up tissue engineering,(14) which starts from the belief that tissue is a cell generated material and thus the direct manipulation and control of cells is of greater importance than the provision of structural, mechanical and chemical cues via the intermediary of a scaffold. This has been termed the "bottom up" or scaffold-free approach to tissue engineering. In order to achieve this cells are formed into clusters, $(15)$ aggregates $(16,17)$ or 2-Dimensional sheets $(18)$. These are then manipulated or positioned with other clusters as "building bricks" or sheets as laminates into 3-Dimensional cellular constructs. This methodology has a number of potential advantages over scaffolds:

- There is no scaffold present so there are no problems of material or degradation product compatibility.

- Cells are cultured in conditions more similar to the 3-Dimensional environment of the body allowing better intercellular communication.

- Clusters made of cells that serve complex functions in organs and are very sensitive to the local environment (e.g hepatocytes) are less likely to redifferentiate and lose function.(15)

However, there are other aspects of tissue engineering where the presence of a scaffold may be beneficial:

- Scaffolds provide mechanical strength while ECM is being generated.

- Assembly of large cellular constructs from sheets or clusters are limited by transport of oxygen, nutrients and waste products until a vascular system develops.

Thus it is likely that a hybrid approach intermediate to the scaffold-directed cell seeded and scaffoldfree organised cellular sub-components approach is likely. This is certainly the case where complex organ like structures are required where the strategy of "let the cells decide" used in the scaffoldfree approach will probably need to be assisted by the use of biochemical cues to direct the desired 
outcome. Thus we move towards the concept of bioprinting, which is essentially an extension of the philosophy that used AM methods to build complex scaffold structures. Bioprinting can be thought of as a combination of the following co-depositions:

- Different types of cells in defined locations.

- Supporting matrix or scaffolds (if required).

- Biochemical cues to control behavior.

This is achieved using printing or AM tools to define their 3-Dimensional arrangement - either topology or providing channels for nutrient access.(19) Although bioprinting has its origins in the area of tissue engineering, and is sometimes described as organ printing,(10) there are other application areas where a printed or artificially fabricated tissue analogue structure is useful including: cell based sensors,(20) drug/toxicity screening (21) and tissue or tumor models.(22)

A key feature of bioprinting is that the deposition process must be cytocompatible as it requires the dispensing of cell containing media. This reduces the range of $A M$ techniques that are feasible because of the need to work in an aqueous or aqueous-gel environment at temperatures from room temperature to $38^{\circ} \mathrm{C}$. Hence most bioprinting work reported in the literature has used filament extrusion, inkjet printing or laser forward transfer as illustrated schematically in figure 1.

\section{Controlling 3D Architectures}

In manufacturing engineering additive manufacture frees the designer from the constraints of moulds and dies reducing dramatically the cost of producing a single, one-off design. It also allows the exploration of design variables. In tissue engineering this enables the easy variation of the internal architecture of a scaffold, allowing the manufacture of a range of structures to allow optimisation, or libraries of structures that can be used in rapid throughput screening for a given application. This has been demonstrated by Melchels et al (23), who used stereolithography (a well established AM method) to produce a range of scaffold designs with different internal architectures and porosity (figure 2) using a biocompatible and degradable polymer. By altering the internal architecture it is possible to vary the specific surface area, scaffold mechanical response to external stress and flow resistance in a bioreactor. Thus it is possible to easily explore the variations in complex internal scaffold architecture as part of an experimental study. Another advantage of architectural control of a porous structure is our ability to better select and control mechanical properties such as stiffness and strength. Conventional tissue scaffolds are fabricated through the foaming of fluid precursors or leaching pore generating particles around which a scaffold has been formed,(1) this produces a fairly random structure when compared with a scaffold fabricated through AM methods (figure 2).

With 3D manufacture, rapid throughput screening of structural variation can be difficult because each structure requires a finite volume of study. However, considerable progress can be made through the use of highly structured surfaces $\left(2 \frac{1}{2}-D\right)$, where micro-manufacturing methods can be used to provide a large range of relatively high aspect ratio structures through varying a small number of structural units or building blocks. This has been used by Unadkat et al who have demonstrated a high throughput method of studying the influence of surface topology on the fate of 
human mesanchymal stem cells.(24) They used conventional silicon lithographic methods to form a master stamp to pattern a thermoplastic biomaterial (poly\{lactic acid\}). The variation in surface toplogy was spatially correlated so by using image analysis techniques they are able to correlate cell response with local topology using optical probes. An example of their so-called topochip is illustrated in figure 3.

As well as allowing the exploration of experimental variables in scaffold design and providing controlled features to study cell interactions, AM produced tissue scaffolds have recently progressed from the laboratory towards clinical application. Two recent studies have demonstrated significant progress. Reichert et al have carried out a large scale trial using a sheep model for hard tissue regeneration in long bones.(25) This compared the performance of a tissue engineering solution, using polycaprolactone/hydroxyapatite composite scaffolds fabricated by a filament deposition method (fused deposition modelling), with an autologous graft. The implant/tissue integration was assessed biomechanically and by X-Ray computed tomography to find that the behaviour of the AM produced implant was equivalent to that of the autograft. Probst et al have reported on the successful outcome of a trial clinical animal model translation study using a designed porous scaffold fabricated by AM.(26) It is highly likely that AM scaffolds will be used clinically in hard tissue reconstructive surgery in the near future, notwithstanding the considerable regulatory hurdles that still need to be overcome.

A key problem in the manufacture of scaffold structures by AM methods is the need to fabricate internal cavities via a layer-by-layer additive process. Such cavities or pore networks are required to assist nutrient transport in a bioreactor and to provide a framework to encourage neovascularisation as ECM is deposited. Self-evidently it is difficult to deposit a material above an empty cavity and this problem is overcome through the use of temporary support structures or through the use of sacrificial materials that are leached away after manufacture to form cavities. $(2,27)$ The use of leaching to form cavities is limited by the need for cytocopatible solvents and leaching products; recent work by Miller et al has proposed a very elegant solution to this problem by developing a soluble carbohydrate glass formulation,(28) that can be readily dispensed by filament microextrusion to form a network that can be leached with water to form a fine network of cylindrical channels (figure 4).

\section{Bioprinting}

Bioprinting has been previously defined as the positioning of biochemicals, biological materials and living cells.(19) It is distinct from AM technologies used to fabricate scaffolds because it allows the deposition of different materials using the same equipment, e.g. the fabrication of structural materials and the simultaneous or sequential deposition of cells.(4) By analogy with colour printing, we can use the technology to print discrete patches of different materials or gradients and transitions between materials to explore their influence in more detail. Printing can be conveniently divided into analogue and digital printing. Analogue printing is normally the direct transfer of an image by some contact process onto a substrate. Common high resolution contact printing technologies used for bioprinting applications include microcontact printing and dip-pen lithography. These have both been used extensively to pattern surfaces with biochemicals and adhesion modifiers, $(29,30)$ chiefly to study how surface features affect the behaviour of cells. Both 
technologies sacrifice speed of application in order to achieve very high spatial resolution, typically $\ll 1 \mu \mathrm{m}$ and neither can be used to deposit cells. Here we will confine our review to computer controlled and digital printing processes. In digital printing the image is converted into pixels and the print consists of a digital pixel on/off application of material. Many forms of digital printing are contactless, the most common is inkjet printing, another digital printing technology, laser forward transfer, has been used extensively for bioprinting applications. The most important computer controlled deposition process is plotting or microextrusion.

\section{Printing Surfaces and Structures to Control the Distribution of Cells}

Inkjet printing is ideally suited to the deposition of biological materials because it is designed for the generation and precise positioning of pl volumes of fluid; (31) these liquid drops can be aqueous solutions and can thus be used for the deposition of most biological species. (19) Inkjet printing was used as a tool for the deposition of cells and biological materials in 1988 by Klebe, who used a Hewlett Packard desktop printer to deposit collagen and fibronectin suspensions as well as cells to form 3-dimensional simple tissue analogue structures. (9) This study used an unmodified printer and simply replaced the graphics ink in a cartridge, after appropriate cleaning, with a biological fluid. Desktop printers, with unmodified ink delivery but with a new ink formulation have been used to deposit functional inorganic materials $(7,8)$ as well as cells and biological materials,(32) illustrating the versatility of this fabrication tool.

The surface resolution attainable by inkjet printing is controlled by the size of a printed drop in flight and its contact angle on the surface after impact.(33) The smallest drops available through inkjet printing are about $1 \mathrm{pl}$ in volume (radius $\approx 6.2 \mu \mathrm{m}$ ) and thus on mildly hydrophilic surfaces have a minimum feature dimension $>10 \mu \mathrm{m}$, similar to that of whole cells. Using electrohydrodynamic drop generation it is possible to produce linear features with around $2 \mu \mathrm{m}$ width.(34) Thus early work featured the use of surface patterning to produce large areas on a surface to selectively control cell adhesion.(35) One advantage of inkjet printing over analogue printing methods is that it is possible to engineer variation in surface concentration through overprinting at different drop densities (figure 5). This has been exploited by the Campbell lab who demonstrated that the response of cells could be directly correlated the surface concentration of printed hormones.(36) They were also able to show that surface concentrations of growth factors are able to control stem cell fate and that different patterns could be used to differentiate cell fate in the same culture dish.(37) These studies used flat polystyrene surfaces for printing and subsequent cell culture. In tissue, cells experience a 3dimensional environment, hence the bioprinting results may not be truly representative. Thus far, fabricating 3-dimensional distributions of growth factors in matrices to probe cell behaviour has not been achieved. However, Campbell has attempted to address some of these issues through using nanofibrous scaffolds onto which growth factors are printed.(38).

\section{Direct Cell Printing}

If the goal of bioprinting is to reproduce tissue structures, it must be able to fabricate complex heterogeneous architectures, either by positioning different cell types in desired locations or by 
inducing progenitor cells to differentiate into the desired type in specific locations. To achieve these tissue analogue or tissue progenitor structures requires a 3-dimensional fabrication facility with multiple material capability in order to position cells within an appropriate temporary structural support, biochemicals with controlled delivery and an open structure to allow the flow of nutrients. (39) Considering that our ability to fabricate and characterise simple, single material, scaffolds is relatively recent the target of a printed tissue is highly ambitious. There are three main technologies that are used for deposition and patterning with cells; these are inkjet printing, $(9,32)$ microextrusion or filament plotting, $(9,40)$ and laser forward transfer.(41) Similar technologies have also been used to transport and place clusters or aggregates of cells.(10) These techniques have similar levels of maximum attainable spatial resolution of approximately $100 \mu \mathrm{m}$. Much higher levels of resolution are attainable using techniques such as electrohydrodynamic jetting, (34) which has been demonstrated with cell suspensions,(42) but considering the typical cell densities used during culture $\left(\approx 10^{7}\right.$ cells $\left./ \mathrm{ml}\right)$ an individual volume of $100 \mathrm{pl}$ (drop radius $\approx 70 \mu \mathrm{m}$ ) will contain on average a single cell and this limits the effective accuracy of deposition for cell placement.

One concern is the level of stress that cells experience during deposition processes. In order to fabricate cell containing structures each method has different requirements for the material surrounding the cell. Inkjet printing requires a low viscosity environment in order to allow efficient drop ejection,(31) microextrusion methods have a very wide range of fluid properties that are compatible with the process, while laser forward transfer requires the cells to be immobilised in a gel.(41) Early reports on inkjet printing cells reported poor viability after deposition,(32) however subsequent work found cell survival rates consistent with unprinted controls, i.e. survival $>95 \%$ with appropriate choice of printing conditions,(43-45) suggesting that the precise formulation of the ink is important. It is noteworthy that Cui's study found that after printing, the cell membrane had a transient increase in density of nanopores, this allowed a greater transfection rate than with unprinted cells and may indicate the mechanism of cell damage that occurs during printing.(45) There has been less systematic study of damage to cells during microextrusion experiments. Chang and Sun have reported cell survival rates in the range of $40-80 \%$ after extrusion of HEPG2 cells with the survival rate decreasing with increasing extrusion pressure.(46) This trend of increasing mechanical stress reducing survival post-deposition is consistent with Saunders' data for cell survival after inkjet printing, where an increase in the actuating voltage used to generate the drops led to a decrease in cell survival.(44) The same trend is seen with laser cell transfer where a greater amount of optical energy is seen to decrease cell survival rates after deposition.(47)

Bioprinting began as a concept for cell manipulation (9)and is now a viable technique for patterning with cells, $(32,40,46)$ and has been combined with a number of biological materials to directly produce hybrid, cell-containing structures. D'Lima et al have recently published a significant result for bioprinting. They used an aqueous solution of polyethyleneglycoldimethacrylate that contained chondrocytes in suspension and printed this into a model osteochondral defect before using a photolytic crosslinker to form a hybrid cell containing hydrogel.(48) After a number of days in culture the printed structure appeared to have integrated into the surrounding tissue (figure 6), demonstrating the feasibility of this approach. Although this study demonstrates great promise, the general use of photoinitiated crosslinking of cell suspensions must address the potential cytotoxicity of the photoinitiator. Williams et al carried out a systematic study of the influence of a number of different photoitiators on cell populations and found that the relative toxicity of each chemical 
studied depended on cell type;(49) hence great care must be taken in selecting appropriate polymer/photoinitiator combinations in the presence of cells.

\section{Prospects for the Future}

We are still a long way from organ printing, although there has been significant advances in the deposition and fabrication technology to allow us to build structures that are analogous to tissue in their composition. However, to develop fully functioning tissue is a much greater step. The use of additive manufacture to produce scaffold structures, mainly for bone tissue engineering, is now almost routine but still only possible with a limited set of biomaterials. The use of AM fabricated scaffolds appear to be making significant steps towards translation, $(25,26)$ and for hard tissue applications the barriers are now regulatory rather than scientific or technical. The prospects are less good for soft connective tissue applications where more compliant materials, e.g. hydrogels are required. Here the main technical challenges are to find suitable biomaterials with appropriate mechanical properties that can be deposited using AM methods. The challenge here is in the field of developing new biomaterials. To date fine detailed hydrogel or biological material scaffolds have been fabricated using indirect methods, where a sacrificial mould is built using AM. $(27,28)$

Bioprinting offers significant promise for the fabrication of structures modelled on tissue architectures. To date the majority of published work is at a relatively low level of technological readiness and has used a very limited range of materials: sodium alginate, modified diblock copolymers and photocured acrylates. The range of available materials is severely constrained by the need to develop cytocompatible gelation mechanisms that can be delivered by AM methods using fluid delivery (inkjet and filament extrusion) and produce a cell containing matrix with an appropriate range of mechanical properties. There is also still considerable uncertainty concerning the level of cell damage that occurs during cell deposition by all bioprinting methods. It is clear that much further work will be needed in this area before regulatory approval can be obtained for translational studies. What is more likely is that we will use these tissue analogue structures for applications such as toxicity screening and drug testing. $(21,39)$ A very interesting development is the use of microfabrication technology to construct tumour models, allowing variation in physiological conditions in vitro.(50)

For any new technology to have an impact in scaffold manufacture or tissue printing, further consideration of generic problems must also be considered. The concept of a scaffold, requires an understanding of the behaviour of an implanted structure within the body an $d$ its interactions at a cellular and tissue scale. The rate of scaffold degradation and ECM formation must be both understood and controllable. However, AM methods provide a significant advance in scaffold design because many of the parameters of architecture that control its physical, mechanical and degradation properties are adjustable within fairly broad limits. Thus, as discussed in detail by Melchels, (24) it is now possible to model the influence of tissue architecture and validate the model by experiments on a range of structures. A challenge here is to completely validate whether designed scaffold structures are reproduced by AM techniques and the recent increase in availability of computed tomography imaging will aid this. Tomography will again assist in monitoring degradation in vitro and possibly functional NMR may be useful in imaging fluid flows and degradation of scaffolds in vivo. Predictive modelling/validation of tissue engineering solutions is 
[Type text]

likely to be the area of immediate impact for these new manufacturing technologies in tissue engineering/regenerative medicine.

\section{REFERENCES}

1. D. W. Hutmacher, Scaffolds in tissue engineering bone and cartilage., Biomaterials 21, 2529-43 (2000).

2. S. J. Hollister, Porous scaffold design for tissue engineering., Nature Mater. 4, 518-24 (2005).

3. M. D. Symes et al., Integrated 3D-printed reactionware for chemical synthesis and analysis., Nature Chem. 4, 349-54 (2012).

4. M. Di Biase, R. E. Saunders, N. Tirelli, B. Derby, Inkjet printing and cell seeding thermoreversible photocurable gel structures, Soft Matter 7, 2639 (2011).

5. E. Malone, H. Lipson, Fab@Home: the personal desktop fabricator kit, Rapid Prototyping J.13, 245-255 (2007).

6. R. Jones et al., RepRap - the replicating rapid prototyper, Robotica 29, 177-191 (2011).

7. M. Mott, J. Song, J. R. G. Evans, Microengineering of Ceramics by Direct Ink-Jet Printing, J. Amer. Ceram. Soc. 58, 1653-1658 (1999).

8. J. Windle, B. Derby, Ink jet printing of PZT aqueous ceramic suspensions, J. Mater. Sci. Lett. 18, 8790 (1999).

9. R. J. Klebe, Cytoscribing: a method for micropositioning cells and the construction of two- and three-dimensional synthetic tissues., Exper. Cell Res. 179, 362-73 (1988).

10. T. Boland, V. Mironov, A. Gutowska, E. a Roth, R. R. Markwald, Cell and organ printing 2: fusion of cell aggregates in three-dimensional gels., Anatom. Rec. Part A, 272, 497-502 (2003).

11. E. Sachlos, J. T. Czernuszka, Making tissue engineering scaffolds work. Review: the application of solid freeform fabrication technology to the production of tissue engineering scaffolds., Europ. Cells Mater. 5, 29-39; discussion 39-40 (2003).

12. M. P. Lutolf, J. a Hubbell, Synthetic biomaterials as instructive extracellular microenvironments for morphogenesis in tissue engineering., Nature Biotech. 23, 47-55 (2005).

13. F. Rosso et al., Smart materials as scaffolds for tissue engineering., J. Cell. Physiol. 203, 465-70 (2005).

14. J. W. Nichol, A. Khademhosseini, Modular Tissue Engineering: Engineering Biological Tissues from the Bottom Up., Soft Matter 5, 1312-1319 (2009).

15. J. M. Kelm, M. Fussenegger, Microscale tissue engineering using gravity-enforced cell assembly., Trends Biotech. 22, 195-202 (2004). 
[Type text]

16. J. M. Kelm et al., Design of custom-shaped vascularized tissues using microtissue spheroids as minimal building units., Tissue Engineering 12, 2151-60 (2006).

17. V. Mironov et al., Organ printing: tissue spheroids as building blocks., Biomaterials 30, 2164-74 (2009).

18. J. Yang et al., Cell sheet engineering : Recreating tissues without biodegradable scaffolds, Biomaterials 26, 6415-22 (2005).

19. B. Derby, Bioprinting: inkjet printing proteins and hybrid cell-containing materials and structures, J. Mater. Chem. 18, 5717 (2008).

20. D. Falconnet, G. Csucs, H. M. Grandin, M. Textor, Surface engineering approaches to micropattern surfaces for cell-based assays., Biomaterials 27, 3044-63 (2006).

21. R. Chang, J. Nam, W. Sun, Direct cell writing of 3D microorgan for in vitro pharmacokinetic model., Tissue Engineering. Part C 14, 157-66 (2008).

22. C. Fischbach, R. Chen, T. Matsumoto, Engineering tumors with 3D scaffolds, Nature Meth. 4, 611 (2007).

23. B. Derby, Inkjet Printing of Functional and Structural Materials: Fluid Property Requirements, Feature Stability, and Resolution, Annual Rev. Mater. Res. 40, 395-414 (2010).

24. F. P. W. Melchels et al., Mathematically defined tissue engineering scaffold architectures prepared by stereolithography., Biomaterials 31, 6909-16 (2010).

25. H. V. Unadkat et al., An algorithm-based topographical biomaterials library to instruct cell fate., PNAS 108, 16565-70 (2011).

26. J. C. Reichert et al., A tissue engineering solution for segmental defect regeneration in loadbearing long bones., Science Transl. Med. 4, 141ra93 (2012).

27. F. A. Probst, D. W. Hutmacher, F. W. Mueller, H. G. Machens, J. T. Schantz, Calvarial reconstruction by customized bioactive implant, Handchirurgie Micrchirurgie Plastische Chirurgie $\mathbf{4 2}$, 369-373 (2010).

28. E. Sachlos, N. Reis, C. Ainsley, B. Derby, J. T. Czernuszka, Novel collagen scaffolds with predefined internal morphology made by solid freeform fabrication., Biomaterials 24, 1487-97 (2003).

29. J. S. Miller et al., Rapid casting of patterned vascular networks for perfusable engineered threedimensional tissues., Nature Mater. 11, 768-774 (2012).

30. C. M. Nelson, C. S. Chen, VE-cadherin simultaneously stimulates and inhibits cell proliferation by altering cytoskeletal structure and tension., J. Cell Sci. 116, 3571-81 (2003).

31. S. Sekula et al., Multiplexed lipid dip-pen nanolithography on subcellular scales for the templating of functional proteins and cell culture., Small 4, 1785-93 (2008).

32. T. Xu, J. Jin, C. Gregory, J. J. Hickman, T. Boland, Inkjet printing of viable mammalian cells., Biomaterials 26, 93-9 (2005). 
33. J. Stringer, B. Derby, Formation and stability of lines produced by inkjet printing., Langmuir 26, 10365-72 (2010).

34. T. Sekitani, Y. Noguchi, U. Zschieschang, H. Klauk, T. Someya, Organic transistors manufactured using inkjet technology with subfemtoliter accuracy., PNAS 105, 4976-80 (2008).

35. E. A. Roth et al., Inkjet printing for high-throughput cell patterning., Biomaterials 25, 3707-15 (2004).

36. P. G. Campbell, E. D. Miller, G. W. Fisher, L. M. Walker, L. E. Weiss, Engineered spatial patterns of FGF-2 immobilized on fibrin direct cell organization., Biomaterials 26, 6762-70 (2005).

37. J. A Phillippi et al., Microenvironments engineered by inkjet bioprinting spatially direct adult stem cells toward muscle- and bone-like subpopulations., Stem Cells 26, 127-34 (2008).

38. E. D. F. Ker et al., Bioprinting of growth factors onto aligned sub-micron fibrous scaffolds for simultaneous control of cell differentiation and alignment., Biomaterials 32, 8097-107 (2011).

39. X. Wang, Y. Yan, R. Zhang, Rapid prototyping as a tool for manufacturing bioartificial livers., Trends Biotech. 25, 505-13 (2007).

40. D. L. Cohen, E. Malone, H. Lipson, L. J. Bonassar, Direct freeform fabrication of seeded hydrogels in arbitrary geometries., Tissue Engineering 12, 1325-35 (2006).

41. J. A. Barron, P. Wu, H. D. Ladouceur, B. R. Ringeisen, Biological laser printing: a novel technique for creating heterogeneous 3-dimensional cell patterns., Biomed. Microdevices 6, 139-47 (2004).

42. S. N. Jayasinghe, A. N. Qureshi, P. a M. Eagles, Electrohydrodynamic jet processing: an advanced electric-field-driven jetting phenomenon for processing living cells., Small 2, 216-9 (2006).

43. M. Nakamura et al., Biocompatible inkjet printing technique for designed seeding of individual living cells., Tissue Engineering 11, 1658-66 (2005).

44. . R. E. Saunders, J. E. Gough, B. Derby, Delivery of human fibroblast cells by piezoelectric dropon-demand inkjet printing., Biomaterials 29, 193-203 (2008).

45. X. Cui, D. Dean, Z. M. Ruggeri, T. Boland, Cell damage evaluation of thermal inkjet printed Chinese hamster ovary cells., Biotech. Bioeng. 106, 963-9 (2010).

46. R. Chang, J. Nam, W. Sun, Effects of dispensing pressure and nozzle diameter on cell survival from solid freeform fabrication-based direct cell writing., Tissue Engineering A 14, 41-8 (2008).

47. S. Catros, B. Guillotin, M. Bačáková, J.-C. Fricain, F. Guillemot, Effect of laser energy, substrate film thickness and bioink viscosity on viability of endothelial cells printed by Laser-Assisted Bioprinting, Appl. Surf. Sci. 257, 5142-5147 (2011).

48. X. Cui, K. Breitenkamp, M. Finn, Direct Human Cartilage Repair Using Three-Dimensional Bioprinting Technology, Tissue Engineering A 18, 1304-1312 (2012). 
[Type text]

49. C. G. Williams, A. N. Malik, T. K. Kim, P. N. Manson, J. H. Elisseeff, Variable cytocompatibility of six cell lines with photoinitiators used for polymerizing hydrogels and cell encapsulation.,

Biomaterials 26, 1211-8 (2005).

50. A. D. Stroock, C. Fischbach, Microfluidic culture models of tumor angiogenesis., Tissue Engineering A 16, 2143-6 (2010). 


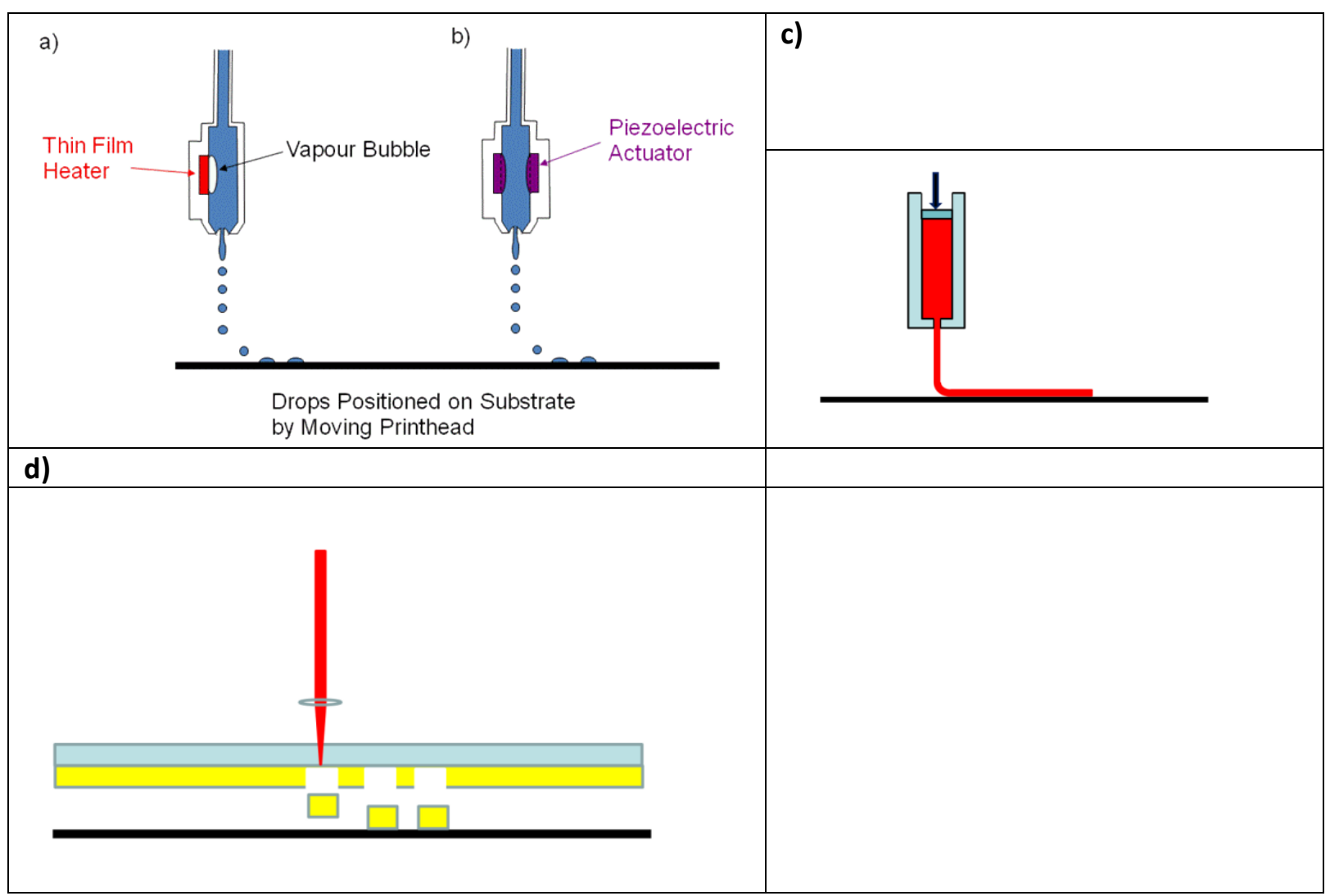

Figure 1 There are a number of Rapid Prototyping/Additive Manufacturing techniques that have been used in bioprinting applications. All produce solids through the sequential deposition of solid layers or slices. Inkjet printing accurately positions drops, which may contain cells in suspension, in the volume range $1-100 \mathrm{pl}$. Drops are generated either by a) heating and vaporizing the ink so that a bubble forces out a liquid drop, or b) by mechanical actuation (reproduced with permission from ref 24). c) Microextrusion or filament plotting deposits a continuous thread of material to build up a layer. d) With laser forward transfer material is initially deposited on a transparent ribbon in gel form. Pulsed laser beam vaporises a small quantity of material, this ejects the remainder of the material illuminated which is transferred to the substrate across a small air gap. 


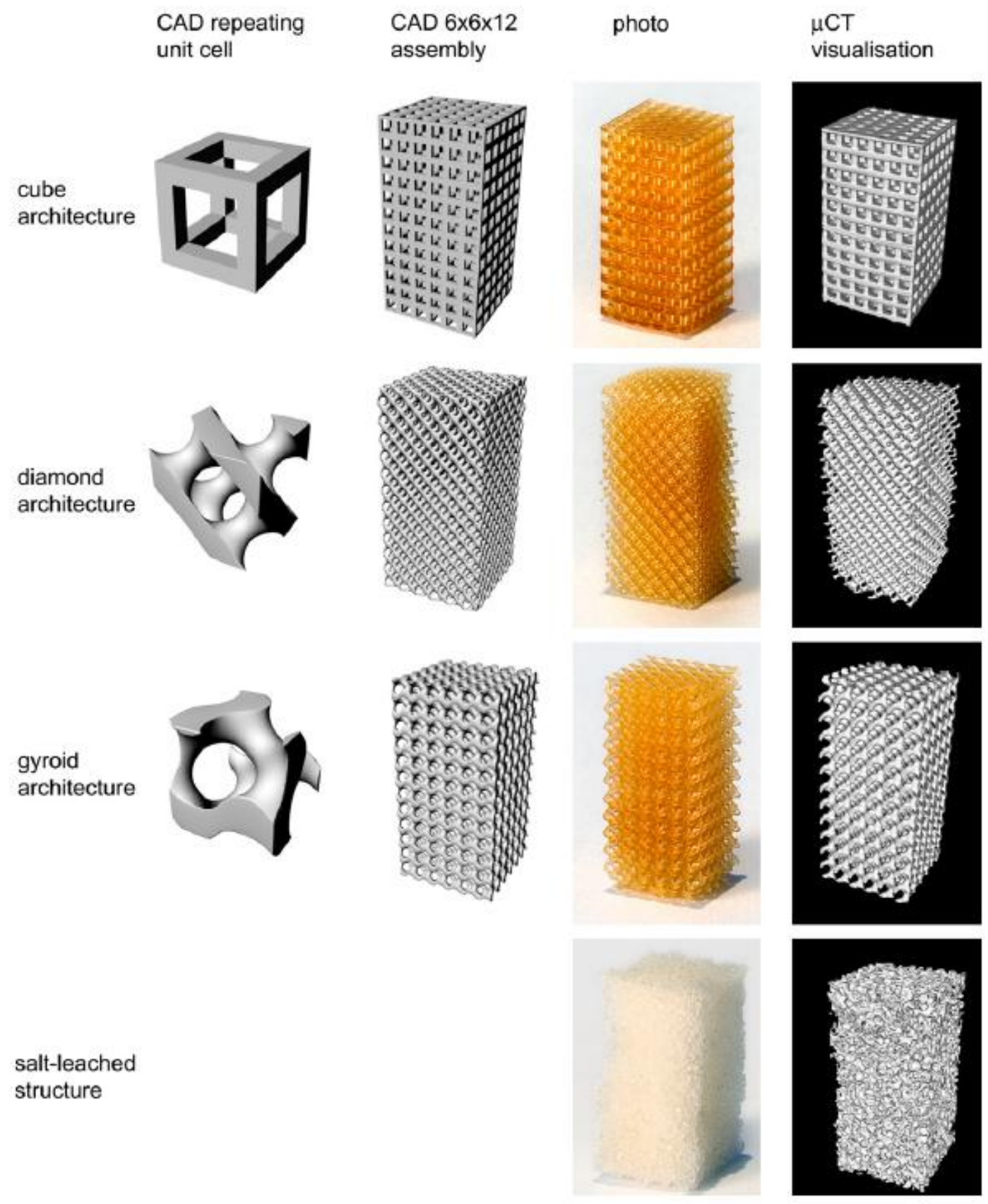

Figure 2 Recent advances in additive manufacturing technology, tomographic reconstruction and numerical modelling methods has allowed workers to design a range of complex internal scaffold architectures with a range of length scales. These can be fabricated with a minimum feature length < $100 \mu \mathrm{m}$ and the resulting structures inspected for perfection. This figure, reproduced with permission from Ref.24, shows three different designed scaffold architectures compared with a structure formed by a random foaming process. 

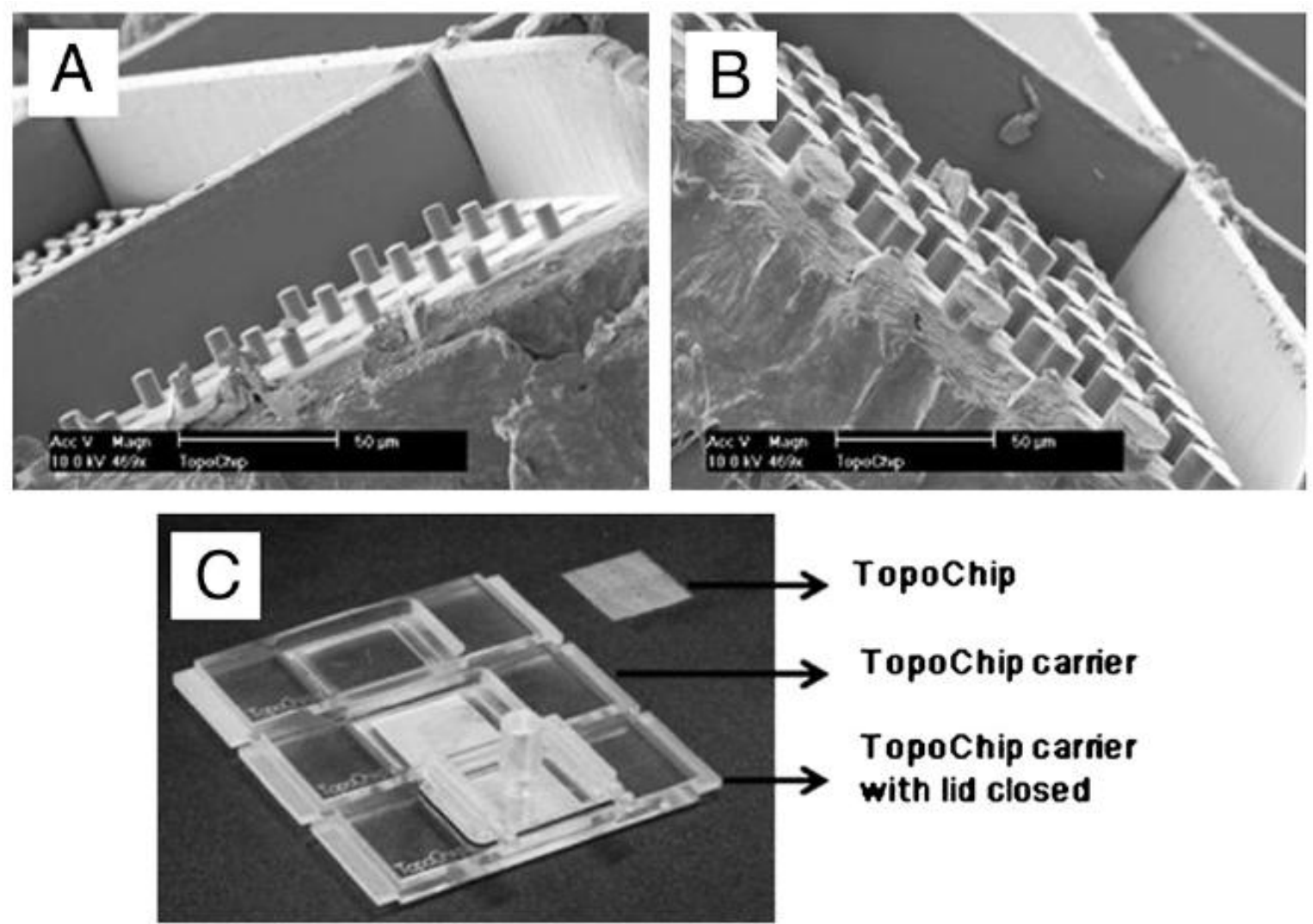

TopoChip

TopoChip carrier

TopoChip carrier

with lid closed

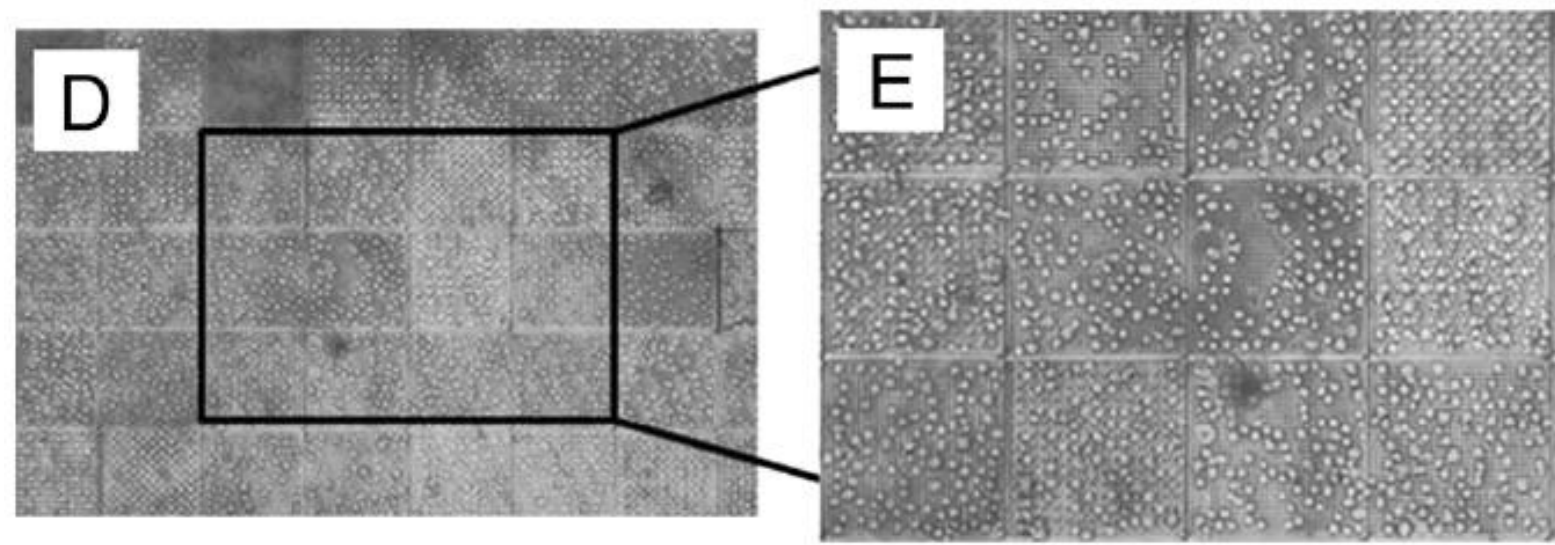

Figure 3 Example of a "topochip" - a method of producing a variation in surface structure that can be identified by spatial correlation. a) and b) show examples of different surface topologies at different locations on the surface (scale bar $=50 \mu \mathrm{m}$ ). c) shows the chip and d) and e) show how local cell behaviour can be identified and correlated to the known position of each surface topology. Reproduced with permission from Ref 25). 


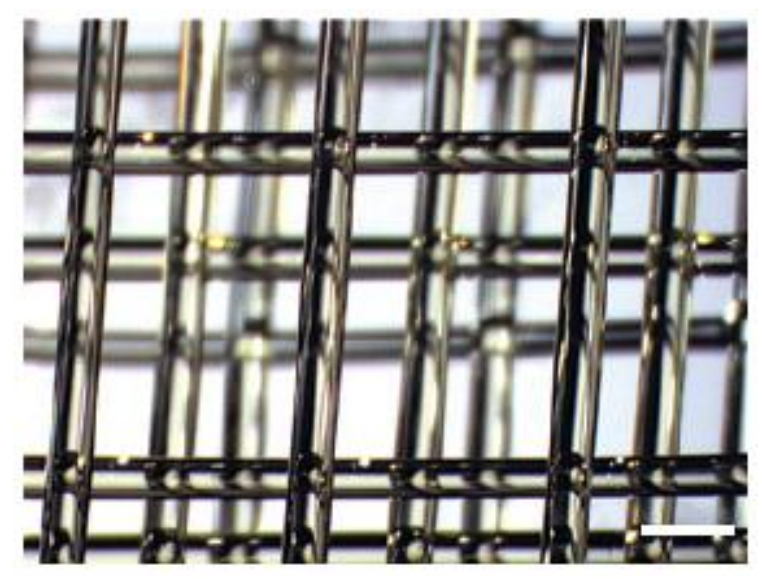

Figure 4 3-Dimensional lattice structure fabricated from a carbohydrate glass structure using a filament extrusion AM technique, reproduced with permission from Ref. 29. A photopolymerisable cell suspension is cast around this structure, which is subsequently removed by leaching to provide a vascular network. Scale bar is $1 \mathrm{~mm}$. 
higher FGF-2 concentration

lower FGF-2 concentration

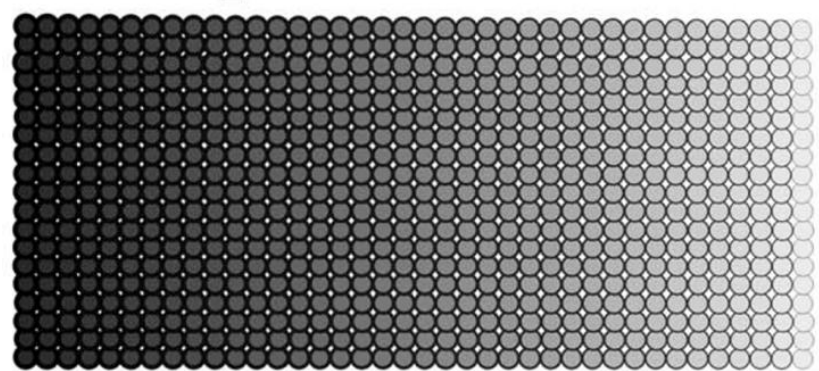

(A)

- multiple overprinted drops

single drop

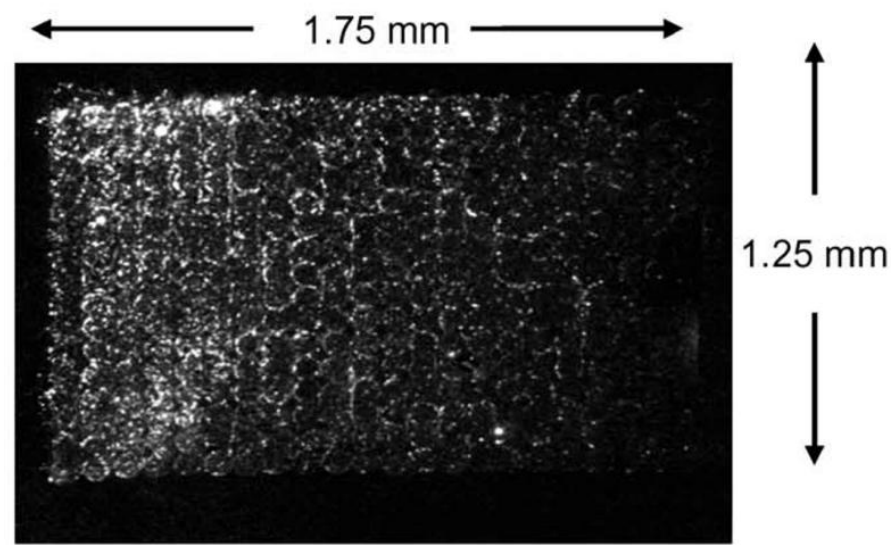

(B)

[FGF-2]

Figure 5 Illustration of how digital printing methods can be used to print spatially defined

concentration gradients of biochemicals. In this example, reproduced with permission from Ref. 36.

A) Schematic of how gradients of fibroblast growth factor-2 (FGF-2) have been printed with different surface concentrations formed by overprinting multiple drops in the same location on the surface. B) Example of an FGF-2 gradient prepared using sequential overprinting of biotinylated FGF-2, which was labeled with streptavidin conjugate quantum dots. 
a)

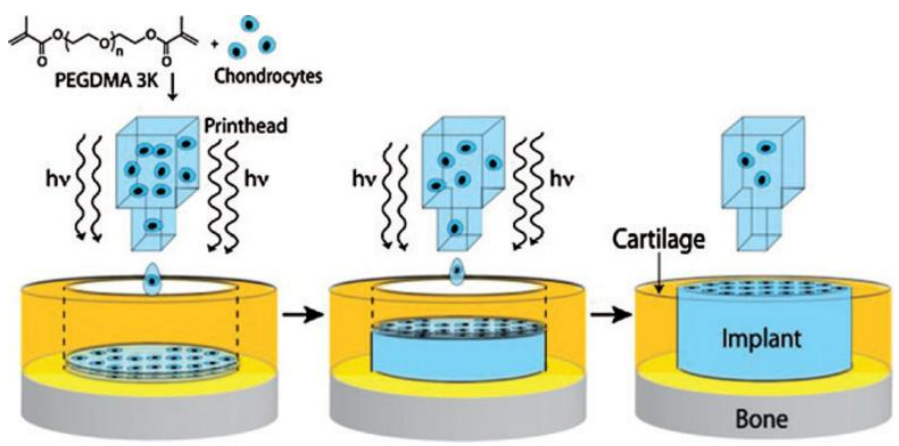

b)

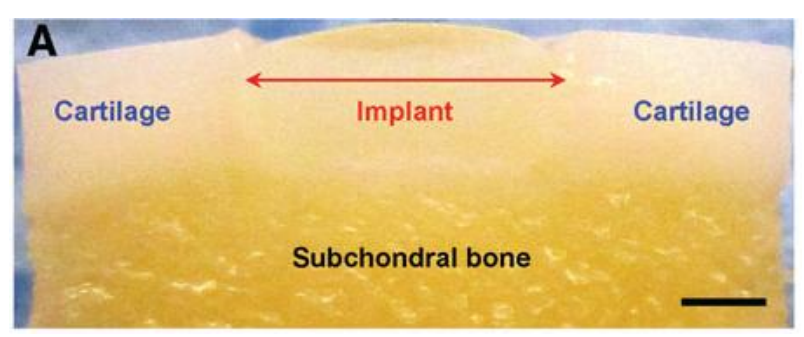

Figure 6 a) Schematic of bioprinting a cartilage analogue structure, combining inkjet printing a PEGDMA, poly(ethylene glycol) dimethacrylate solution containing cells in suspension with a simultaneous photopolymerization process. b) Light microscopy image of cell-containing polyethylene hydrogel printed into a defect formed in an osteochondral plug (scale bar $=2 \mathrm{~mm}$ ) After culture the cells within the printed material express ECM similar to those in the adjacent tissue. Reproduced with permission from Ref. 48. 